\title{
Gzip File Format
}

National Cancer Institute

\section{Source}

National Cancer Institute. gzip File Format. NCI Thesaurus. Code C80220.

A file format consisting of a 10-byte header containing a magic number, a version number, and a timestamp, a Deflate-compressed body, and an 8-byte footer containing a checksum and the length of the original uncompressed data. 